\title{
La formación del profesorado de primaria en educación intercultural: una experiencia a través de la investigación acción
}

\author{
The training of primary school teachers in intercultural education: \\ an experience through action research
}

\author{
Olga González Mediel ${ }^{a}$, Llarela Berríos Valenzuela ${ }^{b}$, Marcela Toro Collantes ${ }^{c}$ \\ ${ }^{a}$ Departamento de Didáctica de la Expresión Musical y Corporal, Universitat de Barcelona, España. \\ ogonzalez@ub.edu \\ ${ }^{b}$ Facultad de Filosofía, Departamento de Educación Parvularia. \\ Universidad Metropolitana de Ciencias de la Educación, Chile. 1larela.berrios@umce.cl \\ ${ }^{c}$ Investigadora independiente, Chile.mltoro@uc.cl
}

\begin{abstract}
La formación del profesorado en educación intercultural es un aspecto esencial de la práctica docente, principalmente en aquellos contextos escolares donde la diversidad cultural está presente en las aulas. Este artículo presenta un estudio, cuyo objetivo general fue crear instancias de formación en educación intercultural para el profesorado de educación primaria. Para este propósito se intervino en un centro educativo público de educación primaria con una alta tasa de alumnado inmigrante, situado en la provincia de Barcelona. De acuerdo a las necesidades e intereses del colegio, se optó por el método de investigación-acción entre los docentes del establecimiento educativo y el equipo investigador. Los resultados permitieron constatar que en un proceso continuo de reflexión y con recursos específicos basados en la interculturalidad, la transformación de la práctica docente permite avanzar hacia el modelo de educación intercultural que fomenta la participación y el diálogo cultural desde el punto de la igualdad.
\end{abstract}

Palabras claves: formación del profesorado, educación inclusiva, educación intercultural, diversidad cultural, investigación cualitativa.

\section{ABSTRACT}

Training teachers in intercultural education has become an essential aspect of teaching practice, mainly in those educational contexts where cultural diversity is present in the classrooms. The present study has been conducted in order to investigate the topic, and its general objective create instances of intercultural education training for primary school teachers. To accomplish this objective, a primary public school with a large number of immigrants, located in the Barcelona province, was intervened. An action research project was conducted based on the needs and interests of the school. The results showed that in continuous and reflexive teaching training with specific resources based on interculturality, teaching practicum transformation moves towards an intercultural education model which fosters participation and cultural dialogue from the standpoint of equality.

Key words: Teacher training, inclusive education, cultural diversity, intercultural education, qualitative research. 


\section{INTRODUCCIÓN}

La educación intercultural, es el modelo educativo recomendado para dar respuesta a la realidad multicultural en las aulas. Sin embargo, el término intercultural puede dar pie a varias interpretaciones, así pues, dedicaremos unos párrafos a conceptualizar el sentido de la palabra intercultural desde una perspectiva descriptiva, sobre todo, para aclarar las diferencias y similitudes con otros términos.

Convenimos con Abdallah-Pretceille (2001), que en el núcleo de los problemas relativos a la interculturalidad encontramos una serie de conceptos como cultura, identidad, etnia, raza y etnicidad, ligados entre sí por complejas relaciones. Los conceptos de cultura y de identidad, forman parte de esta gama de conceptos que, al igual que la libertad y la inteligencia, generan polémica, porque se pueden abordar desde varias perspectivas ideológicas. Asî lo evidencian varios autores que en sus estudios plantean que: pluricultural, intercultural o multicultural tendrán mucho que ver con las diferentes opciones ideológicas que se adopten (Aguirre, 1997; Banks, 2006; Bartolomé, 1997; García, 1993; Soriano, 2001).

Como hay muchos términos que tienen la palabra cultura como base (prefijos y sufijos), el consejo de Europa, a fines de los setenta, precisó la definición de cada uno de ellos y los transcribimos para intentar unificar criterios de conceptualización. Multiculturalidad define la situación de las sociedades, grupos o entidades sociales en las cuales muchos grupos o individuos que pertenecen a diferentes culturas viven juntos (Coelho, 2006; Fernández y Molina, 2005; Malizia, 2007). Pluricultural es casi un sinónimo, indica la existencia de una situación particular, que, en las mismas circunstancias, en lugar de subrayar la existencia de un amplio grupo de culturas en contacto, se destaca únicamente su pluralidad. Transcultural implica movimiento de una situación a otra, e intercultural define un enfoque, procedimiento, proceso dinámico de naturaleza social en el cual los participantes son positivamente impulsados a ser conscientes de su interdependencia y es también una filosofía, política y pensamiento que sintetiza tal enfoque. Las investigaciones de Etxeberría y Elosegui (2010; Mussino y Strozza (2012); Palou (2010); y Sabariego (2002); nos recuerdan que se acepta preferentemente el término de educación intercultural dentro del ámbito educativo europeo, designando el carácter de un proceso educativo dinámico, de interacción, intercambio, apertura y solidaridad efectiva desde el reconocimiento de los valores, de los modos de vida, y de las representaciones simbólicas, tanto dentro de los registros de una misma cultura, como entre culturas diferentes.

Numerosos autores entregan las definiciones, principios, objetivos y características de este modelo educativo que permiten comprenderlo y delimitarlo (Aguado, Gil y Mata, 2005; Bartolomé, 1997; Besalú, 2002; Díaz-Aguado, 2003; Essomba, 1999; Jordán, 1996). La perspectiva intercultural surge como consecuencia de dos o más culturas que coexisten dentro de una misma sociedad y es la culminación de un proceso que empieza en los sesenta, en los Estados Unidos, para dar respuesta a nuevas situaciones de reivindicación étnico-cultural (Banks y Banks, 2007; Baraldi, 2008; Milan, 2007). Sin embargo, desde la interculturalidad crítica para Walsh,

La interculturalidad, en cambio, aún no existe. Es algo por construir. Va mucho más allá del respeto, la tolerancia y el reconocimiento de la diversidad; señala y alienta, más bien, un proceso y proyecto social político dirigido a la construcción de sociedades, relaciones y condiciones de vida nuevas y distintas. (2008, p. 140). 
Desde este punto de vista, la autora se refiere a la cosmovisión de la vida, la cual incluye los saberes, conocimientos, la memoria ancestral, la espiritualidad y la relación con la naturaleza de cada cultura, que coexiste en un determinado país. En esta misma línea Tubino (2004) señala lo siguiente: "la interculturalidad como concepto aparece incorporada en la agenda política de los movimientos indígenas de América Latina. No es un concepto de la academia es parte del reclamo de los indígenas de América Latina”. (p. 1). Y este discurso se debe a la colonización, a la discriminación sistemática de la que han sido objeto los pueblos originarios.

En nuestro contexto más inmediato, la inquietud para dar respuesta en la educación en contextos multiculturales es mucho más reciente, pues es a principios de los años noventa cuando realmente se hace visible Jordán (1996), a pesar de que ya hacía mucho tiempo que España, además de ser un país poli-étnico, también era ya un Estado multinacional.

Consideramos que la educación intercultural alude al conjunto de estudios y prácticas educativas con las cuales se intenta dar respuesta a las cuestiones que plantea la diversidad de grupos étnicos y culturales en una determinada sociedad. Desde la perspectiva de Jordan (2001) "Pretende formar en todos los alumnos, de todos los centros, una competencia cultural madura; es decir, un bagaje de aptitudes y de actitudes que les capacite para funcionar adecuadamente en nuestra sociedad multicultural y multilingüe" (p. 49). Sin embargo, el término "intercultural" expresa la opción de la interacción entre las culturas, lo que enriquece la tarea educativa. Desde otra perspectiva, se afirma que constituye un enfoque, un procedimiento, un proceso dinámico de naturaleza social, en el cual se impulsa positivamente a los participantes a ser conscientes de su interdependencia y es también, una filosofía, una política y un pensamiento que sistematiza la realidad. La denominación de educación intercultural alude a una tendencia reformadora en la práctica educativa (Aguado, Gil y Mata, 2005), y convenimos con Sabariego (2002), que tiene que ser parte integral de un proyecto de construcción social, ello en la medida que se orienta a fomentar el respeto por el pluralismo cultural, y donde la tolerancia no solamente consiste en aceptar pasivamente los derechos de otros grupos culturales, sino que comporta un reconocimiento activo y comprensivo de otras culturas.

Desde este mismo enfoque, de alcance social, también Banks (2006), plantea la educación intercultural como movimiento de reforma escolar, dirigido a incrementar la igualdad educativa de todo el alumnado y que debe conseguir el reconocimiento de múltiples visiones del mundo.

Entendemos que la educación intercultural es una tendencia reformadora en la práctica educativa, que trata de responder a la diversidad cultural de las sociedades actuales. El término, es utilizado con carácter normativo para designar la naturaleza del proceso educativo deseable (Aguado, Gil y Mata, 2005; Bartolomé, 1997; Hajisoteriou y Angelides, 2016). Por otro lado, podemos delimitarla a los programas y prácticas educativas diseñadas e implementadas para mejorar el rendimiento educativo de las poblaciones étnicas y culturales minoritarias y a la vez preparar a los alumnos del grupo mayoritario para aceptar y aprender de las culturas y experiencias de los grupos minoritarios (Banks, 2006).

La educación intercultural se opone al concepto de asimilación, (Besalú, 2002), es decir, se opone a una integración que obligue a deponer y olvidar el propio bagaje cultural y aceptar los autóctonos; en cambio, está a favor de aquella integración que incluye a todos los miembros, cada uno con su propia carga cultural, a la cual no se le pide renunciar. Tampoco es una educación "compensatoria" que identifica "deficiencia" con "diferencia" 
y tiene como único objetivo la incorporación de habilidades en el alumnado recién llegado, sobre todo en materia de capacitación lingüística (pero en un proceso inverso olvidando la cultura originaria de las niñas y niños), sino que aprovecha las diferencias culturales para conocer otras formas de ver y de ser, relacionándolas con las conocidas hasta el momento. En el modelo de educación intercultural, la igualdad y la diversidad son dos caras de la misma moneda y su enfoque práctico al aula tiene presente tanto lo qué se tiene que enseñar como el cómo hacerlo, para favorecer la igualdad y hacer justicia a la diversidad (Biagioli, 2005; Giusti, 2004; Sabariego, 2002). Por tanto, la educación intercultural tiene que tener intención no sólo de apertura, enriquecimiento y construcción mutua, sino de implicación en una sociedad también "intercultural” (Bartolomé, 1997).

En este modelo, es muy importante implicar a toda la comunidad educativa, debido a que este enfoque nos compete a todas y todos, no solamente a las personas que están inmersas en un contexto escolar multicultural (Berríos y Palou, 2014). Entonces, será rol de la escuela ayudar al alumnado a desarrollar el conocimiento, las habilidades y actitudes necesarias para funcionar efectivamente en la cultura comunitaria, en la cultura nacional predominante y con/entre otras culturas y sociedades, lo que podríamos denominar "competencia cultural" (Besalú, 2002).

Tenemos que tener presente que ninguno de los problemas planteados por la diversidad étnica y cultural de la sociedad tiene una solución unilateral. Las medidas educativas son sectoriales dentro de un modelo de sociedad global, pero es desde la escuela y con un modelo como el que pretendemos implantar que podremos abrirnos a las mejoras sociales.

El desafío de la interculturalidad en el ámbito educativo consiste en atender a la diversidad, reconociendo la dimensión personal y cultural del "otro" y de esta forma enriquecer los procesos de enseñanza-aprendizaje, ya que hemos pasado de aulas y centros educativos monoculturales a multiculturales, Malizia (2007). Por esta razón, la formación del profesorado en este ámbito adquiere una relevancia social, la cual se justifica desde dos perspectivas: "la gran presencia de alumnado inmigrante en las aulas y la necesidad de dar una respuesta pedagógica de calidad al reto de construir escuelas inclusivas" (Leiva, 2012, p. 10). Dentro de este contexto es indudable que el profesorado es un elemento clave de la educación intercultural, esto se debe principalmente a que, en el aspecto formativo, éste se convierte en un importante agente de cambio (González, Berríos y Buxarrais, 2013). Por este motivo, convenimos con Leiva (2012), quien indica que la formación intercultural del profesorado es una demanda cada vez mayor en los planes de formación inicial y permanente de las titulaciones de educación. Debido a esto, las facultades de formación del profesorado tienen un rol esencial en la formación inicial y continua en cuanto al desarrollo de conocimientos, competencias, actitudes y didáctica (Escarbajal, 2014; Fiorucci, 2015; Gómez, 2011; Jordan, 1996). La formación debe promover innovaciones curriculares e incorporar contenidos de temas culturales orientados a la diversidad, así como también elaborar y desarrollar nuevas estrategias de enseñanza-aprendizaje que promuevan en el alumnado la reflexión y análisis intercultural. Así pues, este profesorado es el primero que tiene que asumir e integrar las creencias filosóficas, educativas y sociales que comporta un planteamiento intercultural. Sin embargo, es importante reconocer que este profesorado tiene una formación monocultural, lo cual dificulta su capacitación para impartir una educación pluralista y formar a los futuros docentes en este ámbito.

Autores, como Jordán et al. (2004) proponen basar el modelo genérico de formación para el profesorado en tres vertientes: la cognitiva, la técnica pedagógica y la actitudinal. 
Tabla 1. Modelo genérico de formación del profesorado

\begin{tabular}{|l|l|}
\hline Dimensión & Definición \\
\hline Cognitiva & $\begin{array}{l}\text { Esta dimensión se refiere a los conocimientos básicos sobre el entorno } \\
\text { cultural del alumnado inmigrante y a la reflexión crítica de los contenidos } \\
\text { que se deben abordar }\end{array}$ \\
\hline Técnico-Pedagógica & $\begin{array}{l}\text { Se centra en las competencias que le permiten al profesorado desarrollar } \\
\text { estrategias metodológicas y didácticas para poder trabajar la educación } \\
\text { intercultural en el aula. }\end{array}$ \\
\hline Actitudinal & $\begin{array}{l}\text { Basada en la actitud del profesorado hacia el alumnado inmigrante, en esta } \\
\text { dimensión destaca, el proceso reflexivo y crítico-constructivista respecto a } \\
\text { las ideas y prácticas establecidas; replantear la tarea docente hacia una } \\
\text { vertiente más educativa que instructiva. }\end{array}$ \\
\hline
\end{tabular}

Fuente. Adaptación de Jordán et al. (2004).

Para abordar las dimensiones mencionadas, es importante que los docentes tengan presente una serie de orientaciones a considerar, puesto que este enfoque educativo va mucho más allá de una mera transmisión de información, debido a que pide cambiar todo el proceso de enseñanza-aprendizaje, en la línea de cultivar actitudes positivas, (respetar, tolerar, valorar, abrir, empatizar, superar prejuicios y potenciar el sentido crítico), mejorar el autoconcepto del alumnado, (acoger, aceptar, cuidar la seguridad socio-afectiva, reconocer los progresos académicos, incentivar la participación escolar, ayudar a descubrir puntos fuertes e introducir elementos culturales), potenciar la convivencia y la cooperación entre el alumnado, descubrir parecidos culturales, incentivar aprendizajes y juegos cooperativos, conseguir integración socio-afectiva, resolver positivamente los conflictos surgidos, facilitar la comunicación intercultural, desarrollar actividades cívicas, potenciar la igualdad de oportunidades académicas y buscar el máximo rendimiento escolar de todo el alumnado. Para esto, es necesario considerar las diferencias culturales, por lo que se sugiere adaptar las planificaciones curriculares (Cernadas, Santos y Lorenzo, 2013; Jordán, 1996; Jokikokko, 2016; Santos, Cernadas y Lorenzo, 2014).

A continuación, presentamos un esquema que hemos desarrollado, con el fin de reflejar la importancia del profesorado en el proceso de transformación en la educación intercultural. 
Figura 1. Esquema rol del profesorado en educación intercultural

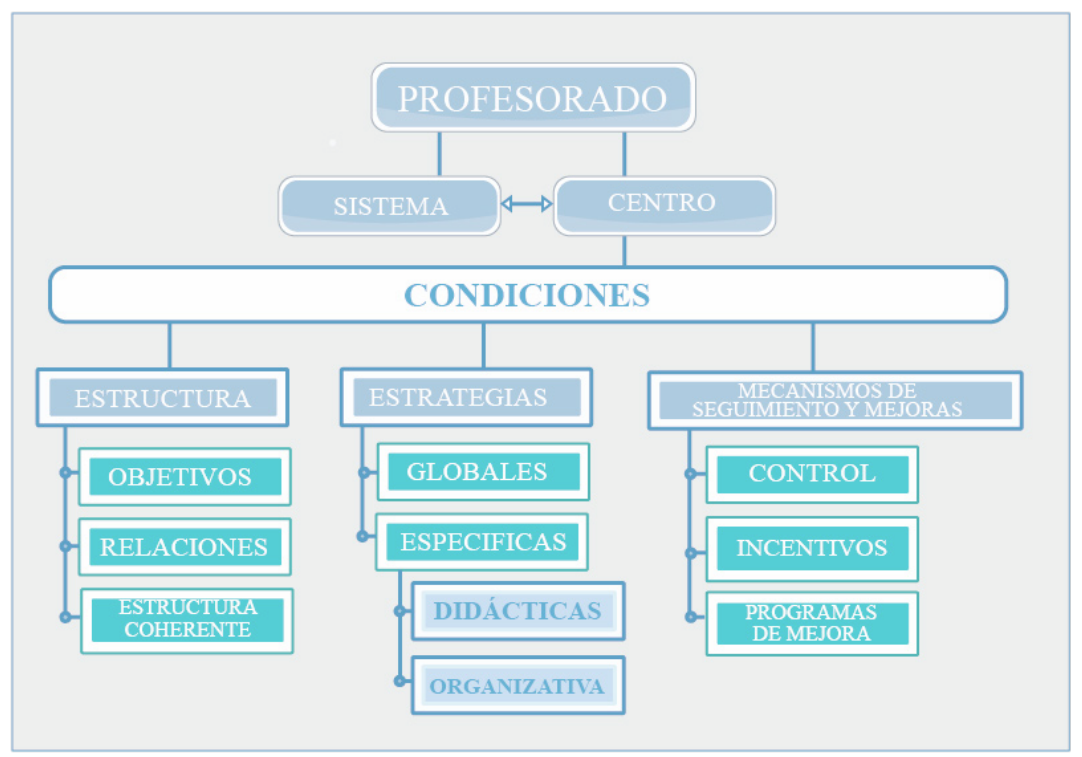

Fuente. Elaboración propia.

\section{METODOLOGÍA}

La metodología empleada para llevar a cabo el estudio se centró en el paradigma cualitativo, basada en el modelo de investigación-acción, la cual, según Bartolomé (1990), consiste en

un modelo de investigar los problemas en educación. Su definición, aunque con variantes en los distintos autores pone el énfasis en el hecho que los investigadores y educadores trabajan juntos en la planificación, implementación y análisis de la investigación que se lleva a cabo para resolver problemas inmediatos y prácticos de los maestros compartiendo la responsabilidad en la toma de decisiones y en la realización de las tareas de investigación (p. 31).

En consideración con lo expuesto anteriormente, este método resulta ser el más idóneo para llevar a cabo la investigación, debido a su vinculación con el objetivo general, que consiste en: crear instancias de formación en educación intercultural para el profesorado de educación primaria. Y los objetivos específicos que surgen del objetivo general son: a) identificar necesidades de educación intercultural del profesorado, b) diseñar una intervención educativa de acuerdo a las necesidades detectadas, c) implementar la intervención con el profesorado, d) evaluar la implementación de la intervención. Las preguntas de investigación que se busca responder en el presente estudio son las siguientes: 1) ¿cómo conseguir transformar la práctica docente apuntando a la educación intercultural? 2) ¿qué tipo de formación para el profesorado en educación intercultural se podrían establecer? 3) ¿qué estrategias metodológicas podríamos utilizar? 
Si bien, el objetivo de la investigación acción se enfoca en el cambio y para ello considera la comprensión y resolución de problemáticas específicas de una entidad social (Bisquerra, 2004; Hernández, Fernández y Baptista, 2014; Latorre, del Rincón y Arnal, 2003; Savin-Baden y Major, 2013). Al respecto Bartolomé y Sandín (2001) señalan que este tipo de metodología se utiliza cuando dos o más instituciones; habitualmente una de ellas orientada a la formación de profesionales y producción científica, mientras que la otra es un colegio o institución en la cual trabajan los profesionales que se pretende formar. Las instituciones deciden asociarse, para resolver juntas las problemáticas concernientes a las prácticas de éstos últimos, articulando los procesos de investigación, innovación, desarrollo y formación profesional.

El contexto en el que se desarrolló la investigación corresponde a un centro educativo de carácter público, que atiende los niveles de educación infantil y primaria en el Distrito de Sant Martí de Provençals de Barcelona. La elección de este centro radica en dos aspectos fundamentales: 1. El compromiso del profesorado para asumir el trabajo de una investigación acción, que respondiera a su formación en educación intercultural; 2. El alumnado inmigrante representa un $14 \%$ de la matrícula total del establecimiento, sin embargo, esta cifra se eleva al $24 \%$ en segundo de primaria, por este motivo, la investigación se focalizó en este nivel educativo. Y es aquí dónde radica la problemática del profesorado, al no contar con una formación en educación intercultural y tener en sus aulas estudiantes de diversos países.

De acuerdo a Kemmis y Mctaggart (1988), este modelo de investigación se realizó en un proceso que se desarrolló en cuatro fases, como se presenta en la siguiente tabla:

Tabla 2. Fases de la investigación-acción

\begin{tabular}{|l|l|}
\hline Fases & Contenido \\
\hline Reflexión inicial & $\begin{array}{l}\text {-Elaboración marco teórico } \\
\text {-Preparación de materiales }\end{array}$ \\
\hline Planificación & $\begin{array}{l}\text {-Diseño del proceso } \\
\text {-Selección de la muestra } \\
\text {-Ajuste de los recursos } \\
\text {-Formación inicial }\end{array}$ \\
\hline Acción & $\begin{array}{l}\text {-Puesta en práctica del plan previsto } \\
\text {-Observación de su funcionamiento }\end{array}$ \\
\hline Reflexión final & $\begin{array}{l}\text {-Análisis de datos } \\
\text {-Interpretación de los datos } \\
\text {-Resultados } \\
\text {-Conclusiones }\end{array}$ \\
\hline
\end{tabular}

Fuente. Adaptada de Kemmis y Mctaggart (1988). 
La investigación cualitativa, responde al grupo de personas, eventos, y sucesos, sobre los cuales se aplicarán las estrategias de recolección de información, sin que necesariamente, sea estadísticamente representativo de la población que se estudia (Bisquerra, 2004; Flick, 2004; Hernández, Fernández y Baptista, 2014; Ruiz, 2012; Savin-Baden y Major, 2013). En este caso, se trata de un muestreo no probabilístico intencional, y estuvo constituida por nueve docentes de educación primaria, de los cuáles tres eran tutores de segundo curso, ya que en ese nivel se llevó a cabo la intervención durante un año académico debido al 24\% de alumnado inmigrante en este nivel. Los otros docentes del centro que participaron son los que realizan las asignaturas de: lenguaje, matemáticas, música, plástica, conocimiento del medio y educación física. Estos docentes fueron incluidos en la investigación debido a que realizan clases en segundo de primaria, y deben estar alineados con los tutores en la formación docente en educación intercultural.

En investigación cualitativa, hoy se acepta que no existe una única forma de conocer y aproximarse a la realidad, explorarla, comprenderla y reconstruirla, y se focaliza en el interés por dar significado e interpretar los acontecimientos desde la perspectiva de las personas implicadas. Debido a esto, se aboga por la pluralidad de estrategias de recolección de información y la adopción de éstas permiten captar la naturaleza abierta, dinámica y flexible de la realidad social y educativa (Bisquerra, 2004; Flick, 2004; Ruiz, 2012; Sandín, 2003). Por este motivo, se previeron utilizar las principales estrategias de recolección de información dentro del modelo de investigación cualitativa (análisis documental, observación, entrevista y diario de sesiones). En la siguiente tabla, se evidencian las técnicas de recogida de la información empleadas en el proceso de investigación.

Tabla 3. Estrategias de recogida de la información

\begin{tabular}{|c|c|c|c|}
\hline & & FASES & \\
\hline \multirow[t]{2}{*}{ ESTRATEGIAS } & $\mathbf{1}^{\mathrm{a}}$ FASE & $2^{\mathrm{a}}$ FASE & $3^{\text {a }}$ FASE \\
\hline & $\begin{array}{l}\text { Análisis del contexto y } \\
\text { planificación del } \\
\text { diseño de intervención }\end{array}$ & $\begin{array}{l}\text { Formación y } \\
\text { transformación }\end{array}$ & $\begin{array}{c}\text { Incidencia y permanencia } \\
\text { del cambio }\end{array}$ \\
\hline \multicolumn{4}{|c|}{ Análisis documental } \\
\hline \multicolumn{4}{|l|}{ Observación } \\
\hline \multicolumn{4}{|l|}{ Diario de sesiones } \\
\hline Entrevistas & & & \\
\hline
\end{tabular}

Fuente. Elaboración propia.

Cabe destacar que para llevar a cabo el plan se acción se dividió en las siguientes etapas: 
Tabla 4. Etapas y actividades plan de acción

\begin{tabular}{|c|c|}
\hline ETAPAS & ACTIVIDADES \\
\hline $\begin{array}{l}\text { Formación del } \\
\text { profesorado en } \\
\text { educación } \\
\text { intercultural }\end{array}$ & $\begin{array}{l}\text {-Aprendizaje sobre la introducción de Educación Intercultural en el } \\
\text { curriculum, a través de recursos didácticos } \\
\text {-Aprendizaje sobre otras culturas } \\
\text {-Aprendizaje sobre metodologías } \\
\text {-Reuniones de ciclo y curso como momentos privilegiados de intercambio } \\
\text { - La tarea del equipo investigador-formador } \\
\text {-La autoformación del profesorado participante }\end{array}$ \\
\hline $\begin{array}{l}\text { Formación en } \\
\text { actividades de } \\
\text { innovación } \\
\text { metodológica }\end{array}$ & $\begin{array}{l}\text { - Trabajo en actividades de innovación curricular intercultural } \\
\text { del profesorado, respecto a la integración del contenido y } \\
\text { logrando los objetivos curriculares } \\
\text { - } \\
\text { Actividades de innovación metodológica del profesorado } \\
\text { para lograr los objetivos propuestos en las estrategias de } \\
\text { enseñanza-aprendizaje } \\
\text { - } \quad \text { Apertura del aula a la familia inmigrante } \\
\text { - } \quad \text { Reacciones del alumnado sobre las actividades desarrolladas: } \\
\text { como las reciben, como las trabajan y que impacto tienen en } \\
\text { los niños/as } \\
\text { - Valoración y opinión personal del profesorado respecto a la } \\
\text { aplicación de la experiencia en el aula, efecto, incidencias, } \\
\text { dificultades, dinámica y clima intercultural. } \\
\text { - } \quad \text { Reflexión y valoración del profesorado al final de esta etapa } \\
\text { - } \quad \text { Daloración general } \\
\text { - }\end{array}$ \\
\hline $\begin{array}{l}\text { Valoración y } \\
\text { opinión del } \\
\text { profesorado } \\
\text { respecto de la } \\
\text { implementación } \\
\text { de la experiencia } \\
\text { en aula }\end{array}$ & $\begin{array}{l}\text { Valoración de la experiencia por parte del profesorado participante } \\
\text {-Impacto sobre el grupo y el alumnado foráneo } \\
\text {-Interés y participación } \\
\text {-Mejora en la integración de los niños y niñas que participan poco } \\
\text {-Protagonismo de los estudiantes foráneos } \\
\text {-Logro de los objetivos curriculares } \\
\text {-Clima del grupo } \\
\text {-Valoración del proceso seguido: desde los recursos al trabajo por áreas } \\
\text {-Dificultades detectadas } \\
\text { - Condiciones } \\
\text {-Propuestas de mejora } \\
\text {-Valoración equipo investigador }\end{array}$ \\
\hline
\end{tabular}

Fuente. Elaboración propia.

El análisis e interpretación de la información es un aspecto esencial en la investigación acción, pues se trata de un proceso cíclico inserto en todas las etapas de la investigación y tiene como propósito contrastar, triangular y validar la información obtenida para establecer las conclusiones. En esta investigación, para el análisis de los datos, se establecieron dos dimensiones: formación del profesorado y educación Intercultural. A partir de 
Estudios Pedagógicos XLVII N 1: 197-217, 2021

LA FORMACIÓN DEL PROFESORADO DE PRIMARIA EN EDUCACIÓN INTERCULTURAL: UNA EXPERIENCIA A TRAVÉS DE LA INVESTIGACIÓN ACCIÓN

estas dimensiones se establecieron tres subdimensiones: a) formación del profesorado en educación intercultural, b) formación del profesorado en actividades de innovación metodológica en educación intercultural y c) valoración y opinión del profesorado respecto a la aplicación de la experiencia en el aula: efectos, incidencia, dificultades, dinámica y clima intercultural. En el proceso de análisis se rescataron los vínculos existentes entre las tres subdimensiones y a partir de ellos se establecieron los resultados. A continuación, se expone el esquema que se utilizó para realizar el análisis de la información.

Figura 2. Esquema del análisis de la información

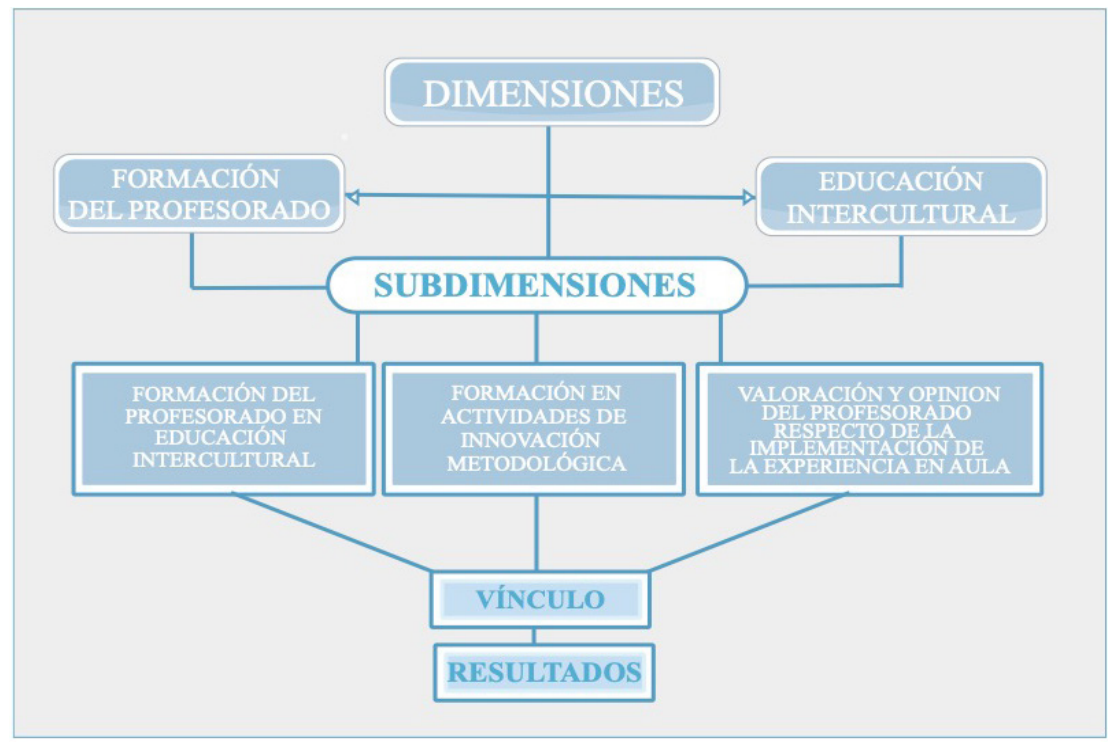

Fuente. Elaboración propia.

La información recolectada a través de las diversas estrategias, se transcribieron de forma íntegra con un procesador de textos (Microsoft Word), y para el procesamiento de la información se utilizó el software QDA (Qualitative Data Analysis) ATLAS.ti, en dos instancias; para la codificación de la información, y en el proceso de organización y estructuración de los datos.

\section{RESULTADOS}

En relación con nuestro objetivo general y con el fin de trabajar en conjunto entre el equipo investigador-formador y el profesorado, se optó por involucrar a los profesores y profesoras de las tres aulas de segundo curso de primaria. De este modo, se pudo trabajar con los mismos contenidos, objetivos y actividades de enseñanza-aprendizaje en todas ellas, facilitando el conocimiento práctico de la idea a desarrollar. 
Con el planteamiento anterior, empezó efectivamente un primer bucle de acción, en el cual se estableció como objetivo básico el "probar" el material elaborado por el equipo investigador, con la intención de validar la posibilidad crear instancias de formación en educación intercultural para el profesorado de educación primaria.

De los resultados obtenidos destaca principalmente el estrecho vínculo entre las dos grandes dimensiones analizadas: formación del profesorado y educación Intercultural. De ellas se establecieron tres subdimensiones, que englobaban todas las categorías de análisis previstas para identificarlas, las cuales se presentan en la siguiente forma:

Tabla 5. Códigos y subdimensiones de análisis

\begin{tabular}{|l|l|}
\hline Códigos & Subdimensiones \\
\hline FPEI & Formación del profesorado en educación intercultural. \\
\hline FPAIEI & $\begin{array}{l}\text { Formación del profesorado en actividades de innovación metodológica en educación } \\
\text { intercultural }\end{array}$ \\
\hline FPEIV & $\begin{array}{l}\text { Valoración y opinión del profesorado respecto a la aplicación de la experiencia en el } \\
\text { aula: efectos, incidencia, dificultades, dinámica y clima intercultural. }\end{array}$ \\
\hline
\end{tabular}

Fuente. Elaboración propia.

Las principales evidencias del proceso de formación del profesorado y transformador de su práctica docente hacia la educación intercultural se encuentran en el análisis de los resultados de las diferentes integraciones de contenidos, como también en los datos que recogen las modificaciones que el profesorado participante ha hecho, respecto a las estrategias metodológicas para conseguirlo. La subdimensión tres recogió, la valoración y opinión del profesorado sobre la aplicación de la experiencia en el aula. El alto número de acotaciones (40), utilizadas para el análisis de las tres subdimensiones, corresponden a las evidencias que se tienen para demostrar el efecto y el impacto de la experiencia desde la perspectiva del profesorado.

El mapa conceptual que se presenta a continuación ayudará a visualizar los resultados del bucle de acción. 


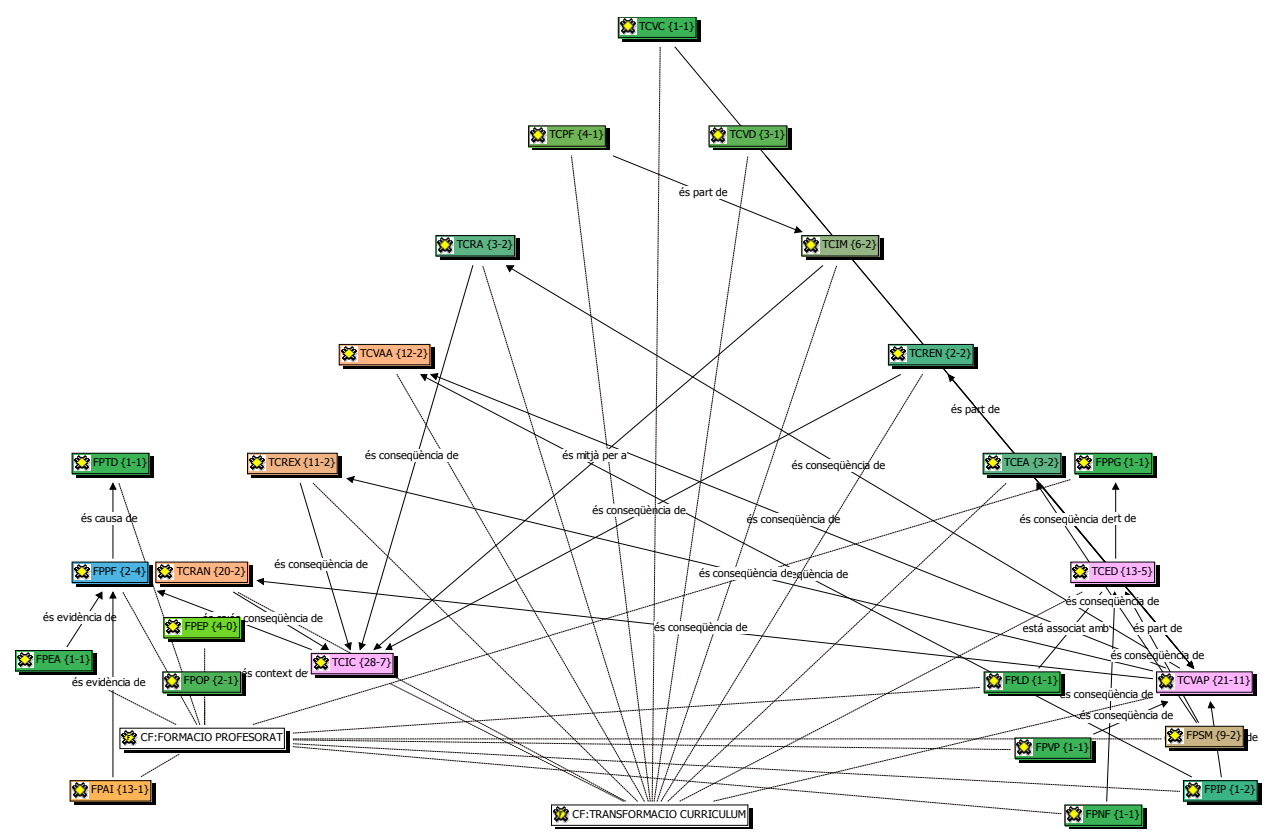

Fuente. Elaboración propia.

\subsection{SUBDIMENSIÓN 1: FORMACIÓN DEL PROFESORADO EN EDUCACIÓN INTERCULTURAL}

En esta subdimensión se recogió, por un lado, lo que se ha realizado versus el modelo de educación intercultural y, por otro lado, hasta qué punto lo que se ha hecho responde a los objetivos de la educación intercultural. Una ventaja en este primer bucle de acción es que el material realizado por el equipo investigador ya estaba validado.

Es importante señalar que el profesorado adoptó este cambio en su práctica educativa y llevó a cabo las actividades propuestas que respondían al modelo de educación intercultural. Efectivamente, se evidenció en el análisis, que los tres maestros tutores y los especialistas de cada área han realizado su trabajo respetando las indicaciones de las unidades de programación, preparadas de forma conjunta por el equipo investigador para las diversas áreas del conocimiento: matemáticas, plástica, música, lenguaje, educación física y conocimiento del medio, cada uno en diferentes momentos y con diversas metodologías de trabajo.

Las primeras sesiones se desarrollaron de forma parecida en las tres aulas y todo el profesorado trabajó en los contenidos previstos, aunque con cierta flexibilidad horaria en una franja educativa, algún tutor/a organizó varias actividades aprovechando toda la tarde y/o toda la mañana para dar continuidad a la temática propuesta, mientras que otros respetaron las materias programadas, en tanto los especialistas (música y educación física), lo hicieron en el momento que les tocaba tener a cada grupo. Cabe señalar que los tres cursos lograron los objetivos previstos en esta fase. Aunque ya ha quedado patente que 
el profesorado ha llevado a cabo la integración de contenido prevista para todas las áreas, tenemos que triangular la información y demostrar que todo lo que indican que han hecho, efectivamente se ha cumplido, por lo cual exponemos algunos fragmentos de los registros narrativos, pertenecientes a algunas de las sesiones de trabajo.

En primer lugar, se reparten las hojas con la letra de una canción en portugués y lápices colores por mesas, la profesora tutora de $2 A$, explica a niños y niñas que tendrán que subrayar en diferentes colores las palabras de la canción que sean iguales al castellano, en otro color las que sean iguales al catalán, y no se deben subrayar las que no se asemejen de ni uno de los idiomas... Antes de empezar la actividad, todos los niños tienen claro que su compañero Joao es de Brasil, donde se habla portugués, y han cantado la canción en la hora de música. (Registro de observación 7: Actividad-Brasil, Curso 2A)

Ahora La tutora de 2 C explica (señalando el mapa de Peters), que está en blanco porque irán buscando e indicando los países que conocerán a lo largo del curso...A continuación, reparten una copia del mapa de Peters a cada alumno, para que los niños y niñas busquen, marquen y pinten: Cataluña, España y Brasil., y una vez realizo esto el mapa se guarda en la carpeta de conocimiento del medio hasta una próxima sesión. (Registro de observación, 17: Actividad-Brasil, Curso 2C)

Además de la consecución de los objetivos planteados para todas las áreas, es importante destacar que el propio alumnado ha ampliado el abanico de posibilidades de integración de contenido cultural puesto que, desde una metodología participativa, los mismos niños y niñas, tanto los nacidos en Brasil como los nacidos en España y en los otros países existentes en el aula, han buscado y encontrado parecidos en los cuentos, han pedido información extra sobre el país y se han dado cuenta claramente de los parecidos y diferencias entre las lenguas (castellano, catalán y portugués).

\subsection{SUBDIMENSIÓN 2: FORMACIÓN DEL PROFESORADO EN ACTIVIDADES DE INNOVACIÓN METODOLÓGICA EN EDUCACIÓN INTERCULTURAL}

Un indicador claro de cambio en la práctica educativa del profesorado participante es que no sólo incorporaron nuevos contenidos en el currículum, sino que también fueron capaces de adaptar nuevas estrategias y recursos para conseguir los objetivos interculturales.

Desde el comienzo, el profesorado participante adoptó iniciativas muy interesantes respecto a su propia práctica educativa. Destacan, en primer lugar, y muy ligado a las directrices de un modelo educativo intercultural, el dar voz a las personas representantes de las culturas trabajadas en clases, y esto ha servido también para modificar las estrategias de enseñanza-aprendizaje pasando el profesor a ser un "conductor" y el alumno recién llegado adopta el rol de "especialista cultural". Lo anteriormente, descrito se evidencia en los siguientes relatos:

Se le pregunta a una niña del curso sobre Ecuador. lo localiza al mapa con la ayuda de Miguel y Mao (ecuatorianos) que sí que lo sabían. Luego localizan Brasil y Cataluña. (Registro de observación 27, Actividad Ecuador, Curso 2B) 
Estudios Pedagógicos XLVII N 1: 197-217, 2021

LA FORMACIÓN DEL PROFESORADO DE PRIMARIA EN EDUCACIÓN INTERCULTURAL: UNA EXPERIENCIA A TRAVÉS DE LA INVESTIGACIÓN ACCIÓN

El alumno brasileño ayuda a pronunciar bien algunas palabras en portugués que aparecen en la canción. Y después la cantamos todos juntos. Cuesta un poco que aprendan algunas partes, pero finalmente ayudados el compañero brasileño y la docente lo logran. (Registro de observación 20, Actividad: Brasil Curso 2A)

En general se evidencia que las sesiones se han presentado de manera participativa y lúdica, la metodología es muy diferente a la del trabajo con el libro de texto, tal y como se evidencia en el fragmento:

La sesión se planteó de manera participativa y como un juego sobre un mapa de Peters se debe calcular y comprobar cuántas veces cabía la silueta de España dentro de Ecuador. (Registro de observación 30, Actividad Educador, Curso 2B)

Como ejemplo del tipo de introducción cultural que queríamos hacer, reflejamos la manera de introducir las sesiones, desde la música, con la intención de darnos cuenta de que queríamos no sólo trabajar una canción de otro país (objetivo curricular), sino que queríamos hacerlo porque era, en este caso, de Brasil, el país donde nacieron nuestros compañeros y/o compañeras.

¡Hoy cantaremos una canción muy bonita que viene de un país muy especial, del Brasil! El país donde nació nuestro compañero y amigo (nombre del niño) ... (Registro de observación 25, Actividad Brasil, Curso 2A)

Por su parte, los profesores tutores introdujeron elementos y actividades que no estaban previstas y que han colaborado en las sesiones: animaron al especialista de educación física a realizar juegos populares de los diversos países del aula. También una idea muy interesante, consistió en que una alumna de Brasil contará el cuento de la "Caperucita roja" en portugués, donde, aprovechando los parecidos entre los idiomas, se consiguió que el alumnado se diera cuenta desde la práctica, sobre las semejanzas entre los idiomas, lo que dio paso a la capacitación metalingüística.

Una modificación importante en la práctica educativa, y muy en línea con las indicaciones de abrir el currículum a la participación de las familias, fue la de invitar a las familias de los diversos países presentes en el aula (Argentina, Brasil, Chile, China, Ecuador, Marruecos y México), donde las familias enseñaron parte de su cultura a los niños a través de canciones, bailes y comida típica.

Finalmente, es importante destacar un ejemplo de innovación "extraescolar" que la escuela tenía programada con anterioridad a nuestra intervención. Dicha actividad iba en la línea del currículum intercultural y al momento de trabajar la cultura de Brasil, el profesorado del centro animó a las familias brasileñas de la escuela para promover la semana de la comida típica de dicho país, organizando diversas actividades en el comedor del centro educativo. 


\subsection{SUBDIMENSIÓN 3: VALORACIÓNY OPINIÓN DEL PROFESORADO RESPECTOA LAAPLICACIÓN} DE LA EXPERIENCIA EN EL AULA: EFECTOS, INCIDENCIA, DIFICULTADES, DINÁMICA Y CLIMA INTERCULTURAL

Respecto a la valoración y opinión del profesorado respecto a la experiencia en el aula, los profesores han demostrado un alto grado de satisfacción después de las sesiones de trabajo, todos coinciden en valorar positivamente la experiencia, destacando la alta participación del alumnado, y la promoción de un clima de aula colaborador y motivador, como reflejan los siguientes párrafos de ejemplo. En el siguiente comentario de una de las profesoras, hace mención a una de las características más interesantes a tener presente, la cual se repetirá a lo largo de toda la experiencia, se trata de la naturalidad con la que el alumnado recibe la información de las diversas culturas.

El nivel de participación ha sido muy alto, los niños se interesan mucho por todo que lo que les puedas explicar de otros países. Además, hay muchos niños que tienen conocimientos de diferentes países del mundo, lo viven con total naturalidad y de forma muy positiva y alentadora. (Entrevistada 10)

Destacamos el comentario de un profesor que demuestra el impacto que se ha visto más allá de la experiencia de segundo de primaria y que justifica el trabajo en esta línea.

Creo que ante el éxito de la experiencia de Brasil todos los alumnos tienen ganas de participar y de que le toque a su país lo antes posible. Incluso alumnos de otros cursos. Ahora se sienten más seguros y ven el uso que hacemos del material, esto complace e interesa a todo el mundo. (Entrevistado, 13)

Sobre la valoración general, la visión global de la experiencia les ha parecido positiva, (corroborando lo que el resto de los documentos recogidos nos indicaban).

Como profesor trabajar en un colegio donde hay una fuerte presencia de alumnado inmigrante, es un desafío constante en el tema de educación intercultural y como llevarla cabo. A través de este trabajo que realizamos en conjunto con un equipo investigador, valoro de forma positiva el hecho de reflexionar sobre la práctica docente que realizamos e implementar material innovador para trabajar la interculturalidad en las aulas, la experiencia ha sido beneficiosa tanto para nosotros como para los niños. (Entrevistado, 14)

Positiva y mejorable a medida que vamos probando y conversando sobre las diferentes culturas y países a trabajar. Así que bien, pienso que ha sido una experiencia muy positiva y un pequeño descubrimiento que favorece el aprendizaje significativo y ayuda en el clima intercultural dentro del aula. (Entrevistado, 11)

Ha sido una experiencia enriquecedora como profesora, ahora si pienso que es complejo reflexionar sobre la práctica de lo que uno hace día a día, pero el hecho de tener el espacio para hacerlo, trabajar con un equipo investigador, proponer actividades y elementos que favorezcan la interculturalidad en el aula y hacerlo con los niños, para 
Estudios Pedagógicos XLVII N 1: 197-217, 2021

LA FORMACIÓN DEL PROFESORADO DE PRIMARIA EN EDUCACIÓN INTERCULTURAL: UNA EXPERIENCIA A

TRAVÉS DE LA INVESTIGACIÓN ACCIÓN

mí ha sido gratificante, he aprendido sobre educación intercultural y la naturalidad con que ellos viven la interculturalidad sorprende, realmente debemos mantener esto en el colegio, porqué tenemos muchos alumnos inmigrantes y de países muy diversos. (Entrevistada, 12)

Es muy importante, en el proceso de reflexión final, detectar las dificultades surgidas, a fin de poder solucionarlas antes de plantear un nuevo bucle de acción. Las dificultades surgidas se han presentado en los siguientes aspectos: metodologías y tiempo de dedicación. Resultó difícil dar respuesta a la primera de ellas, ya que todo el profesorado ha estado de acuerdo en señalar que las actividades y metodologías de trabajo, se salen del esquema habitual, por tanto, han generado más desorden que una clase "convencional" y que el alumnado estaba un poco más ansioso, pero era debido al entusiasmo generado por las nuevas actividades. La solución a esto se logró en el transcurso de la investigación, puesto que, la novedad de las actividades ya no eran un elemento definitorio de las siguientes sesiones.

La dificultad del tiempo de dedicación se encuentra en los límites de tipo técnico, inherente a la carga curricular, y alude al tema de la flexibilidad horaria que tiene el profesorado para desarrollar las actividades, ya que se debe ocupar un tiempo considerable para poder articular las diversas materias en relación a la cultura y país tratado.

\section{DISCUSIÓN Y CONCLUSIONES}

En base a los resultados analizados anteriormente y en relación con los objetivos planteados en la presente investigación, se pueden establecer las siguientes conclusiones: De acuerdo con el objetivo general: Crear instancias de formación en educación intercultural para el profesorado de educación primaria y al contrastarlo con los resultados del estudio, se aprecia que pudimos dar respuesta a la pregunta que nos planteamos ¿cómo conseguir transformar la práctica docente apuntando a la educación intercultural?, se evidenció que la práctica docente del profesorado implicado en la investigación, no sólo tuvo un carácter técnico, sino que fue más allá, debido a que dio paso a una práctica reflexiva, intelectual y autónoma. Considerando estos aspectos, llegamos a la investigación acción (Bisquerra, 2004; Bartolomé y Sandín, 2001). Cabe destacar que efectivamente, el profesorado incorporó cambios en su práctica educativa y realizó actividades que respondían al modelo de educación intercultural, permitiendo así transformar la práctica pedagógica a partir de un modelo de formación intercultural e inclusivo, el cual propicia el cambio y la mejora, poniendo en práctica estrategias de participación y toma de decisiones en forma democrática. Sin duda, la investigación-acción, supuso una metodológica muy adecuada para promover la reflexión, la formación y el cambio. El compromiso y la implicación del profesorado durante toda la investigación, ha sido clave para lograr el conocimiento del modelo de educación intercultural y cómo ejecutar un proceso de transformación desde el enfoque intercultural transversal, que además impactó en el currículum, por lo tanto, aquí respondemos a la segunda pregunta de la investigación ¿qué tipo de formación para el profesorado en educación intercultural se podría establecer? En este caso, se estableció una formación dinámica, activa y basada en la reflexión, la crítica, la experiencia y la praxis del cuerpo docente apoyado por el equipo de investigación, la cual se vio facilitada 
por la realidad multicultural al interior de las aulas. Prueba de lo anterior, es el alto grado de satisfacción expuesto por el profesorado en relación con las experiencias de aula, el aprendizaje logrado al conocer y aplicar nuevas metodologías de trabajo y material innovador, los cuales fortalecen su formación en el tema. Un aspecto destacable, fue la alta participación de los niños y niñas en las diversas actividades, así como la promoción de un clima de aula colaborador, incluyente y motivador. Esto va en la línea de (López y Pérez, 2013; García y Arroyo, 2014), quienes señalan que la Educación Intercultural requiere dar respuesta a la multiculturalidad del alumnado y para ello es necesario realizar un cambio en la Formación del Profesorado, debido al rol educativo y social que desempeñan. En este sentido, convenimos con (Besalú, 2002) al indicar que "educación intercultural no se hace con leyes, normas, teorías y declaraciones, sino que sólo será posible y real con los profesionales y de aquí la importancia de su formación y de su compromiso" (p. 237), sobre la base de la práctica docente. Esto es lo que ha realizado el equipo docente (tutores y profesores de asignatura), por lo tanto, el tipo de formación del profesorado en educación intercultural que se puede establecer es aquel que, si bien aborda el discurso teórico, también considera primordial la práctica de éste, para favorecer la transformación en la escuela. Desde esta perspectiva, el proyecto educativo del centro educativo se tiene que vincular con la interculturalidad y la multiculturalidad, con el fin de favorecer la formación docente en este ámbito. Tal y como se evidenció en este caso, sólo si el proyecto es vivenciado por los docentes, cobra sentido para generar un ambiente favorable y que contribuya a su formación. Por otro lado, es importante indicar que los docentes valoren y respeten las diversas culturas en el proceso de interacción en el ámbito escolar. Esto se inicia con la sensibilización del profesorado en el tema.

Para continuar avanzando en la construcción de la formación docente en educación intercultural, es necesario que se promuevan las competencias pertinentes. Una formación desde la dimensión intercultural tiene que considerar la riqueza de los pueblos culturales diversos, así como también debe contribuir a eliminar toda clase de prejuicios culturales. Por otro lado, la elaboración de estrategias metodológicas y recursos didácticos, deben potenciar la reflexión y el análisis sobre el currículum, el cual debe tener la capacidad de integrar las asignaturas y tratar transversalmente los temas interculturalidad de forma crítica.

Respecto a la tercera pregunta que guío la investigación ¿qué estrategias metodológicas se podrían utilizar? tal y como señalan varios de los autores consultados (Besalú, 2002; Díaz-Aguado, 2003; D’Antoni, 2016; Lamberti, 2010; Palaiogou y Dimitriadou, 2013), en educación intercultural es necesario adoptar innovaciones didácticas, mediante las cuales se pudiera concretar el objetivo: aprendizaje-cooperativo. Se evidenció que, en este aspecto, el profesorado logró un cambio en su práctica educativa, a través de diversos elementos metodológicos:

- Innovación en el planteamiento de las estrategias de enseñanza-aprendizaje, lo cual promovió el aprendizaje significativo del alumnado desde la perspectiva intercultural.

- Estrategias de tipo cooperativo (equipo investigador-formativo y profesorado).

- Búsqueda exhaustiva de material, elaboración y aplicación de recursos. 
Si bien, cada profesor/a utilizó diversas estrategias metodológicas, se evidenció que todas ellas fueron efectivas para el logro de los objetivos propuestos. Aunque esto no estuvo exento de dificultades, ya que las actividades y metodologías se salían del esquema habitual utilizado por los docentes, por tanto, generaron en las primeras sesiones más desorden que una clase "convencional" en el aula, debido que los niños y niñas se encontraban ansiosos. Esto ocurrió producto del entusiasmo generado por las nuevas actividades. La solución a esto se logró en el transcurso de la investigación, puesto que la novedad de las actividades ya no eran un elemento definitorio de las siguientes sesiones. Por otro lado, también se detectó como dificultad el tiempo de dedicación, el cual se encuentra en los límites de orden técnico, es inherente a la carga curricular, y alude al tema de la flexibilidad horaria que tiene el profesorado para desarrollar las actividades, ya que se debe ocupar un tiempo considerable para poder articular las diversas materias en relación a la cultura y país tratado. Más allá de esto, cabe destacar que, durante todo el proceso investigador, se produjo un intercambio de ideas, estrategias, recursos y materiales entre el profesorado y el equipo investigador. incorporando también al alumnado y familias. Para la incorporación de las familias en el proceso de transformación, se consideraron las recomendaciones de Torres (2007); Jordán (2009); Sánchez y García (2009); Santos y Lorenzo (2009); Baker y Clark (2010); Leiva (2011); Hüpping y Büker (2014). Podemos decir que efectivamente se logró la participación de las familias inmigrantes del aula (Argentina, Brasil, Chile, China, Ecuador, Marruecos y México), debido a que se les otorgó la oportunidad de colaborar activamente, aportando el conocimiento de su cultura en las diversas actividades interculturales de la escuela. En ese contexto las familias enseñaron parte de su cultura a los niños/as mediante canciones, bailes y comida típica. Esta actividad no sólo potenció la incorporación y participación de las familias, sino que, además sirvió para que la comunidad educativa conociera y valorar las otras culturas desde una perspectiva de inclusión y respeto por la diversidad cultural.

En relación con el alumnado, se promovió el conocimiento de diversas culturas, a través de las cuales se pudo establecer las semejanzas y diferencias con la cultura de acogida. Se estableció un diálogo cultural desde la igualdad, considerando la diversidad étnica cultural como un elemento positivo para la convivencia. Esto de acuerdo a lo establecido en el modelo de educación intercultural (Bartolomé, 1997; Jordán et al., 2004; Banks, 2006; Palou, 2010; Fiorucci y Catarci, 2015). En esta línea, se considera que el alumnado ha conseguido desarrollar competencias interculturales en la naturalidad del contexto de la escuela (Bravo, 2011; Catarci, 2013; Gil, 2008). La afirmación se basa principalmente, en el interés del alumnado por conocer otras culturas, su alta participación e implicación en cada una de las actividades desarrolladas, la satisfacción y el aprendizaje significativo, que ha marcado todo el proceso de investigación. Por otro lado, se consiguió la valoración de los niños y niñas nacidos en otros países. En este aspecto, cobra importancia el hecho de dar voz y otorgar protagonismo a las actividades relacionadas con su país de origen, lo que permitió estimular la autoestima de forma positiva y contribuir en la formación de su identidad personal. (Harris, Jamison y Trujillo, 2008; Soriano y Franco, 2010). El alumnado inmigrante demostró una mejora en la participación de su grupo de clases y se implicó constantemente en el desarrollo de las actividades durante todo el proceso investigador.

El hecho de elaborar una serie de recursos desde la perspectiva intercultural, ajustados a las necesidades e intereses del centro educativo, ha permitido una sinergia entre el profesorado y el equipo investigador, lo cual impactó directamente en el currículum y aula, contribuyendo a la aproximación de un modelo educativo intercultural. 


\section{REFERENCIAS BIBLIOGRÁFICAS}

Abdallah-Pretceille, M. (2001). La educación intercultural. Barcelona: Idea-Books.

Aguirre, A. (1997). Cultura e identidad cultural. Barcelona: Bardenas.

Aguado, T., Gil, I. y Mata, P. (2005). Educación intercultural: una propuesta para la transformación de la escuela. Madrid: Los libros de la Catarata.

Baker, T. \& Clark, J. (2010). Cooperative learning - a double-edged sword: a cooperative learning model for use with diverse student groups. Intercultural Education, 21(3), 257-268. doi: $10.1080 / 14675981003760440$

Banks, J. (2006). Race, culture and education: The selected works of James A. Banks. New York: Routledge.

Banks, J. \& Banks, C. (eds.) (2007). Multicultural education: Issues and perspectives. Hoboken. New York: Wiley/Jossey-Bass Education.

Baraldi, C. (2008). Comunicazione interculturale e diversità. Roma: Carocci.

Bartolomé, M. y Anguera, M. (coords.) (1990). La investigación cooperativa: Una vía para la innovación en la Universidad. Barcelona: PPU.

Bartolomé, M. (coord.) (1997). Diagnóstico a la escuela multicultural. Barcelona: Cedecs.

Bartolomé, M. y Sandín, M. (2001). Metodologia Qualitativa en Educació. Departament

d'Investigació i Diagnòstic en Educació. Barcelona: Universitat de Barcelona.

Berríos, LL. y Palou, B. (2014). Educación intercultural en Chile: la integración del alumnado extranjero en el sistema escolar. Educación y Educadores, 17(3), 405-426. doi:10.5294/ edu.2014.17.3.1.

Besalú, X. (2002). Diversidad cultural y educación. Madrid: Síntesis.

Biagioli, R. (2005). Educare all'interculturalità. Teorie, modelli, esperienze scolastiche. Milano: Franco Angeli.

Bisquerra, R. (coord.) (2004). Metodología de la investigación educativa. Madrid: La Muralla.

Bravo, J. (2011). La Competencia Intercultural en Alumnado de Educación Primaria: Diseño y evaluación de un plan de intervención para su desarrollo (tesis doctoral). Universidad de Alicante, España.

Catarci, M. (2013). Interculturalim in Italian primary schools with a high concentration of immigrant students. Intercultural Education, 24(5), 456-475. doi:10.1080/14675986.2013.827946

Cernadas, F., Santos, M. y Lorenzo, M. (2013). Los profesores ante la educación intercultural: el desafío de la formación sobre el terreno. Revista de Investigación Educativa, 31(2), 555-570. doi:10.6018/rie.31.2.155391

Coelho, E. (2006). Enseñar y aprender en escuelas multiculturales. Una aproximación integrada. Barcelona: Horsori Editorial, S.L.

D’ Antoni, M. (2016). Interculturalidad: juego y metodologías de aula de primaria. Revista Electrónica Actualidades Investigativas en Educación, 16(3), 1-19. doi:10.15517/aie.v16i3.26067

Díaz-Aguado, M. (2003). Educación intercultural y aprendizaje cooperativo. Madrid: Ediciones Pirámide.

Escarbajal, A. (2014). La educación intercultural en los centros educativos. Revista Electrónica Interuniversitaria de Formación del Profesorado, 17(2), 29-43. doi:10.6018/reifop.17.2.197291

Essomba, M. (coord.) (1999). Construir la escuela intercultural. Reflexiones y propuestas para trabajar la diversidad étnica y cultural. Barcelona: Graó.

Etxeberría, F. y Elosegui, K. (2010). Integración del alumnado inmigrante. Revista Española de Educación Comparada, 16(2010), 235-263. doi: 10.5944/ree.16.2010.7531

Fernández, T. y Molina, J. (Coords.). (2005). Multiculturalidad y educación, teorías, ámbitos, prácticas. Madrid: Alianza Editorial, S.A.

Fiorucci, M. (2015). La formazione interculturale degli insegnanti e degli educatori. Formazione \& Insegnamento, XIII(1), 55-69. doi: 107346/-fei-XIII-01-15_05 
Estudios Pedagógicos XLVII N 1: 197-217, 2021

LA FORMACIÓN DEL PROFESORADO DE PRIMARIA EN EDUCACIÓN INTERCULTURAL: UNA EXPERIENCIA A

TRAVÉS DE LA INVESTIGACIÓN ACCIÓN

Fiorucci, M. \& Catarci, M. (2015). Il mondo a scuola. Per un'educazione interculturale. Roma: Edizioni Conoscenza.

Flick, U. (2004). Introducción a la investigación cualitativa. Madrid: Ediciones Morata S.L.

García, F. (1993). La educación multicultural y el concepto de cultura. Revista de Educación, 302, 83-111.

García, L. y Arroyo, M. (2014). La formación del profesorado en Educación Intercultural: un repaso sobre su formación inicial y permanente. Revista de Educación Inclusiva, 7(2), 127-142.

Gil, I. (2008). El enfoque intercultural en la educación primaria: una mirada a la práctica escolar (tesis doctoral). Universidad Nacional de Educación a Distancia, Madrid, España.

Giusti, M. (2004). Pedagogia interculturale. Teorie, metodologia, laboratori. Roma-Bari: Editori Laterza.

Gómez, I. (2011). La competencia intercultural en la formación inicial de los maestros de educación infantil en la universidad de Castilla la Mancha (tesis doctoral). Universidad Nacional de Educación a Distancia, Madrid, España.

González, O., Berríos, LL. y Buxarrais, M. (2013). La sensibilidad del profesorado hacia el modelo de educación intercultural: Necesidades, situación actual y propuesta de un instrumento de medida. Estudios Pedagógicos, 39(2), 147-164. doi:10.4067/S0718-07052013000200010

Hajisoteriou, C. \& Angelides, P. (2016). The globalisation of intercultural education. The politics of macro-micro integration. London: Palgrave-Macmillan.

Harris, L., Jamison, K. \& Trujillo, M. (2008). Disparities in the educational success of immigrants: An assessment of the immigrant effect for Asians and Latinos. The ANNALS of the American Academy of Political and Social Science, 620(1), 90-114.

Hernández, R., Fernández, C. y Baptista, P. (2014). Metodología de la investigación. $6^{a}$ edición. México D.F: Mc Graw Hill Education.

Hüpping, B. \& Büker, P. (2014). The development of intercultural pedagogy and its influences on primary school: conclusions and perspectives. Intercultural Education, 25(1), 1-13. doi:10.1080/14675986.2014.878072

Jokikokko, K. (2016). Reframing teachers' intercultural learning as an emotional process. Intercultural Education 27(3), 217-230. doi: 10.1080/14675986.2016.1150648

Jordán, J. (1996). Propuestas de educación intercultural para profesores. Barcelona: CEAC S.A.

Jordán, J. (coord.) (2001). La educación intercultural, una respuesta a tiempo. Barcelona: EDIUOC.

Jordán, J., Besalú, X., Bartolomé, M., Aguado, T., Moreno, C. y Sanz, M. (2004). La formación del profesorado en educación intercultural. Madrid: Los Libros de la Catarata.

Jordán, J. (2009). Hacia una relación de partenariado entre profesores y familias inmigrantes. Revista Complutense de Educación, 20(1), 79-97. Recuperado de https://core.ac.uk/download/ pdf/38820712.pdf

Kemmis, S. \& Mctaggart, R. (1988). Cómo planificar la Investigación-acción, Barcelona: Laertes.

Lamberti, S. (2010). Apprendimento Cooperativo e Educazione Interculturale: Percorsi e Attività per la Scuola Primaria. Trento: Erickson.

Latorre, A., del Rincón, D. y Arnal, J. (2003). Bases Metodológicas de la Investigación Educativa. Barcelona: Ediciones Experiencia.

Leiva, J. (2011). La participación de las familias inmigrantes en la escuela intercultural: un estudio cualitativo. Educatio Siglo XXI, 29(2), 389-416.

Leiva, J. (2012). La formación en educación intercultural del profesorado y la comunidad educativa. Revista Electrónica de Investigación y Docencia (REID), Número Monográfico, 8-31. Recuperado de http://www.ujaen.es/revista/reid/monografico/n2/REIDM2art1.pdf

López, E. y Pérez, E. (2013). Formación permanente del profesorado y práctica docente intercultural: contenidos actitudinales y complementariedad competencial. Espiral. Cuadernos del profesorado, 6(12), 32-42. Recuperado de http://www.cepcuevasolula.es/espiral/ articulos/ ESPIRALVOL_6_N_12_ART_3.pdf 
Malizia, P. (2007). Da "Mono" a "Multi". Dieci temi sulla società multiculturale. Milano: Franco Angeli.

Milan, G. (2007). Comprendere e costruire l'intercultura. Lecce: Pensa Multimedia.

Mussino, E. \& Strozza, S. (2012). The delayed school progress of the children of immigrants in lower secondary education in Italy. Journal of Ethnic and Migration Studies, 38(1), 41-57. doi: 10.1080/1369183X.2012.640014

Palaiologou, N. \& Dimitriadou, C. (2013). Multicultural/Intercultural Education Issues in Preservice Teacher Education Courses: The Case of Greece. Multicultural Education Review, 5(2), 49-84. doi: 10.1080/2005615X.2013.11102902

Palou, B. (2010). La integración de la juventud de origen magrebí en Cataluña (tesis doctoral). Universitat de Barcelona, España.

Ruiz, J. (2012). Metodología de la investigación cualitativa. 5a edición. Bilbao: Deusto.

Sabariego, M. (2002). La educación intercultural ante los retos del siglo XXI, Bilbao: Desclée De Brouwer.

Sánchez, C. y García, A. (2009). Implicación de las familias en una escuela intercultural: una visión compartida. Revista de Educación Inclusiva, 2(2). 159-185.

Sandín, M. (2003). Investigación cualitativa en educación: Fundamentos y tradiciones. Madrid: McGraw-Hill.

Santos, M. y Lorenzo, M. (2009). La participación de las familias inmigrantes en la escuela. Un estudio centrado en la procedencia. Revista de Educación, 350(4), 277-300.

Santos, A., Cernadas, F. y Lorenzo, M. (2014). La inclusión educativa de la inmigración y la formación intercultural del profesorado. Revista Electrónica Interuniversitaria de Formación del Profesorado, 17(2), 123-137. doi:10.6018/reifop.17.2.196931

Savin-Baden, M. \& Major, C. (2013). Qualitative Research: The essential guide to theory and practice. London: Routledge.

Soriano, E. (2001). Identidad cultural y ciudadanía intercultural: su contexto educativo. Madrid: La Muralla.

Soriano, E. y Franco, C. (2010). Mejora de la autoestima y de la competencia emocional en adolescentes inmigrantes sudamericanos residentes en España a través de un programa psicoeducativo de Mindfulness (conciencia plena). Revista de Investigación Educativa, 28(2), 297-312.

Torres, J. (2007). Centros escolares y familias en sociedades multiculturales. Revista Andalucía educativa. Interculturalidad en la escuela, 60, 24-27.

Tubino, F. (2004). En el Perú hay interculturalidad a nivel de discurso. Entrevista de Arturo Quispe Lázaro. Revista Electrónica Construyendo Nuestra Interculturalidad, 1(1), 1-6. http://www. interculturalidad.org/numero01/a/arti/a_pun_010404b-

Walsh, C. (2008). Interculturalidad, plurinacionalidad y decolonialidad: las insurgencias políticoepistémicas de refundar el Estado". Tabula Rasa, (9), 131-152. 
